\title{
Charmed Pseudoscalar and Vector Mesons: a Comprehensive QCD Sum-Rule View of Their Decay Constants
}

\author{
Wolfgang Lucha ${ }^{1, a}$, Dmitri Melikhov ${ }^{2,3, b}$, and Silvano Simula $a^{4, c}$ \\ ${ }^{1}$ Institute for High Energy Physics, Austrian Academy of Sciences, Nikolsdorfergasse 18, A-1050 Vienna, \\ Austria \\ ${ }^{2}$ Faculty of Physics, University of Vienna, Boltzmanngasse 5, A-1090 Vienna, Austria \\ ${ }^{3}$ D. V. Skobeltsyn Institute of Nuclear Physics, M. V. Lomonosov Moscow State University, 119991, Moscow, \\ Russia \\ ${ }^{4}$ INFN, Sezione di Roma Tre, Via della Vasca Navale 84, I-00146, Roma, Italy
}

\begin{abstract}
In spite of undeniable similarities of the applied techniques, somewhat different challenges are encountered when extracting, from QCD sum rules derived from two-point correlators of appropriate interpolating heavy-light quark currents, the decay constants of charmed mesons of pseudoscalar nature, on the one hand, or of vector nature, on the other hand. This observation justifies a rather careful reassessment of the corresponding results.
\end{abstract}

\section{Introduction: QCD sum rules in a nutshell-techniques and applications}

QCD sum rules [1] are relations between features of hadrons- the bound states governed by the strong interactions - and the parameters of their underlying quantum field theory-QCD. Such relations may be established (rather straightforwardly) by analyzing vacuum expectation values of nonlocal products of interpolating operators - specifically, of appropriate quark currents - at both QCD and hadron level. Upon application of Wilson's operator product expansion (OPE) for casting, at QCD level, any arising nonlocal operator product into the form of a series of local operators, contributions of both perturbative as well as nonperturbative (NP) origin enter: the former are usually represented by dispersion integrals of certain spectral densities while the latter-also called the "power" corrections-involve the vacuum expectation values of all local OPE operators-crucial quantities going, in this context, under the name of "vacuum condensates." Then, performing a Borel transformation from one's momentum variable to a new variable, the Borel parameter $\tau$, lessens the importance of hadronic excited and continuum states for such "Borelized" sum rules and removes potential subtraction terms. Our lack of knowledge about higher states is dealt with by postulating quark-hadron duality: all contributions of hadron excited and continuum states roughly cancel against those of perturbative QCD above an effective threshold $s_{\text {eff }}(\tau)$.

Here, after sketching, in Sect. 2, the QCD sum-rule extraction of heavy-meson decay constants and recalling, in Sect. 3, a few ideas for improvement of this concept, we focus to its intrinsic uncertainties. Its systematic errors are subject to at least two effects demanding our attention: an optimal perturbative behaviour, discussed in Sect. 4, and the fake impact of the renormalization scale, $\mu$, analyzed in Sect. 5.

\footnotetext{
ae-mail: Wolfgang.Lucha@oeaw.ac.at

be-mail: dmitri_melikhov@gmx.de

ce-mail: simula@roma3.infn.it
} 


\section{Charmed pseudoscalar $\left(D_{(s)}\right)$ and vector $\left(D_{(s)}^{*}\right)$ meson decay constants}

In order to predict the decay constants $f_{\mathrm{P}, \mathrm{V}}$ of charmed pseudoscalar $(\mathrm{P})$ and vector $(\mathrm{V})$ mesons of mass $M_{\mathrm{P}, \mathrm{V}}$ considered as bound states of a charmed quark $c$ of mass $m_{c}$ and a light quark $q=d, s$ of mass $m_{q}$, we use two-point correlators of appropriate currents, given in terms of the QCD degrees of freedom, to find QCD sum rules involving both spectral densities $\rho^{(\mathrm{P}, \mathrm{V})}(s, \mu)$ and nonperturbative terms $\Pi_{\mathrm{NP}}^{(\mathrm{P}, \mathrm{V})}(\tau, \mu)$ at the relevant renormalization scale(s) $\mu$. Pseudoscalar currents then yield, for pseudoscalar mesons $\mathrm{P}$,

$$
f_{\mathrm{P}}^{2} M_{\mathrm{P}}^{4} \exp \left(-M_{\mathrm{P}}^{2} \tau\right)=\int_{\left(m_{c}+m_{q}\right)^{2}}^{s_{\mathrm{eff}}(\tau)} \mathrm{d} s \mathrm{e}^{-s \tau} \rho^{(\mathrm{P})}(s, \mu)+\Pi_{\mathrm{NP}}^{(\mathrm{P})}(\tau, \mu) \equiv \widetilde{\Pi}_{\mathrm{P}}\left(\tau, s_{\mathrm{eff}}(\tau)\right),
$$

which suggests to define dual masses and dual decay constants via the dual correlator $\widetilde{\Pi}_{\mathrm{P}}\left(\tau, s_{\mathrm{eff}}(\tau)\right)$ by

$$
M_{\text {dual }}^{2}(\tau) \equiv-\frac{\mathrm{d}}{\mathrm{d} \tau} \log \widetilde{\Pi}_{\mathrm{P}}\left(\tau, s_{\mathrm{eff}}(\tau)\right), \quad f_{\text {dual }}^{2}(\tau) \equiv \frac{\exp \left(M_{\mathrm{P}}^{2} \tau\right)}{M_{\mathrm{P}}^{4}} \widetilde{\Pi}_{\mathrm{P}}\left(\tau, s_{\mathrm{eff}}(\tau)\right)
$$

Starting, however, from vector currents yields the counterparts of Eqs. (1) and (2) for vector mesons V:

$$
\begin{aligned}
& f_{\mathrm{V}}^{2} M_{\mathrm{V}}^{2} \exp \left(-M_{\mathrm{V}}^{2} \tau\right)=\int_{\left(m_{c}+m_{q}\right)^{2}}^{s_{\mathrm{eff}}(\tau)} \mathrm{d} s \mathrm{e}^{-s \tau} \rho^{(\mathrm{V})}(s, \mu)+\Pi_{\mathrm{NP}}^{(\mathrm{V})}(\tau, \mu) \equiv \widetilde{\Pi}_{\mathrm{V}}\left(\tau, s_{\mathrm{eff}}(\tau)\right), \\
& M_{\text {dual }}^{2}(\tau) \equiv-\frac{\mathrm{d}}{\mathrm{d} \tau} \log \widetilde{\Pi}_{\mathrm{V}}\left(\tau, s_{\mathrm{eff}}(\tau)\right), \quad f_{\text {dual }}^{2}(\tau) \equiv \frac{\exp \left(M_{\mathrm{V}}^{2} \tau\right)}{M_{\mathrm{V}}^{2}} \widetilde{\Pi}_{\mathrm{V}}\left(\tau, s_{\mathrm{eff}}(\tau)\right) .
\end{aligned}
$$

For our OPE input required at QCD level, we use the rather standard set of parameter values in Table 1.

Table 1. Numerical parameter values employed as input to the charmed-meson operator product expansions.

\begin{tabular}{cc}
\hline \hline Quantity & Numerical input value \\
\hline $\bar{m}_{d}(2 \mathrm{GeV})$ & $(3.42 \pm 0.09) \mathrm{MeV}$ \\
$\bar{m}_{s}(2 \mathrm{GeV})$ & $(93.8 \pm 2.4) \mathrm{MeV}$ \\
$\bar{m}_{c}\left(\bar{m}_{c}\right)$ & $(1275 \pm 25) \mathrm{MeV}$ \\
$\alpha_{\mathrm{s}}\left(M_{Z}\right)$ & $0.1184 \pm 0.0020$ \\
$\langle\bar{q} q\rangle(2 \mathrm{GeV})$ & $-[(267 \pm 17) \mathrm{MeV}]^{3}$ \\
$\langle\bar{s} s\rangle(2 \mathrm{GeV})$ & $(0.8 \pm 0.3) \times\langle\bar{q} q\rangle(2 \mathrm{GeV})$ \\
$\left\langle\frac{\alpha_{\mathrm{s}}}{\pi} G G\right\rangle$ & $(0.024 \pm 0.012) \mathrm{GeV}^{4}$ \\
\hline \hline
\end{tabular}

\section{Improving QCD sum rules by advanced extraction of hadronic properties}

The accuracy of QCD sum-rule predictions for hadronic observables extracted by rather long-standing traditional techniques [1] may be significantly improved by dropping the requirement of Borel stability [2-6] — reflecting merely the prejudice that the value of any such observable at its extremum in $\tau$ forms a good approximation to its actual value - and the perhaps very naïve belief that the effective threshold at QCD level does not know about $\tau$ [7-11]: Earlier analyses [2-6] (backed up by quantum mechanics, 
where exact solutions may be derived by just solving Schrödinger equations) forced us to conclude that predictions relying on Borel stability may emerge rather far from the truth and that effective thresholds will depend on $\tau$; they culminated in a simple prescription [7-11] for the extraction of hadron features:

- The admissible $\tau$ range is determined by requiring, at the lower end, the ground-state contribution to be sufficiently large and, at the upper end, the power-correction contributions to be reasonably small. For the charmed pseudoscalar and vector mesons, the demands may be satisfied if choosing as Borel windows $0.1 \mathrm{GeV}^{-2}<\tau<0.5 \mathrm{GeV}^{-2}$ for $D, D^{*}, D_{s}^{*}$ or $0.1 \mathrm{GeV}^{-2}<\tau<0.6 \mathrm{GeV}^{-2}$ for $D_{s}$ [12-14].

- The functional dependence of the threshold $s_{\text {eff }}(\tau)$ on $\tau$ is modelled by adopting a power-law Ansatz,

$$
s_{\mathrm{eff}}^{(n)}(\tau)=\sum_{j=0}^{n} s_{j} \tau^{j},
$$

with expansion coefficients $s_{j}$ determined by minimizing, over a set of $N$ equidistant discrete points, $\tau_{i}$, in the allowable $\tau$ range, the deviation of the predicted from the measured meson masses squared:

$$
\chi^{2} \equiv \frac{1}{N} \sum_{i=1}^{N}\left[M_{\mathrm{dual}}^{2}\left(\tau_{i}\right)-M_{\mathrm{P}, \mathrm{V}}^{2}\right]^{2} .
$$

- Remembering a lesson drawn from quantum-mechanical analogues of QCD sum rules, the spread of results found for the order $n=1,2,3$ of the Ansatz (5) is taken as hint of the intrinsic sum-rule error.
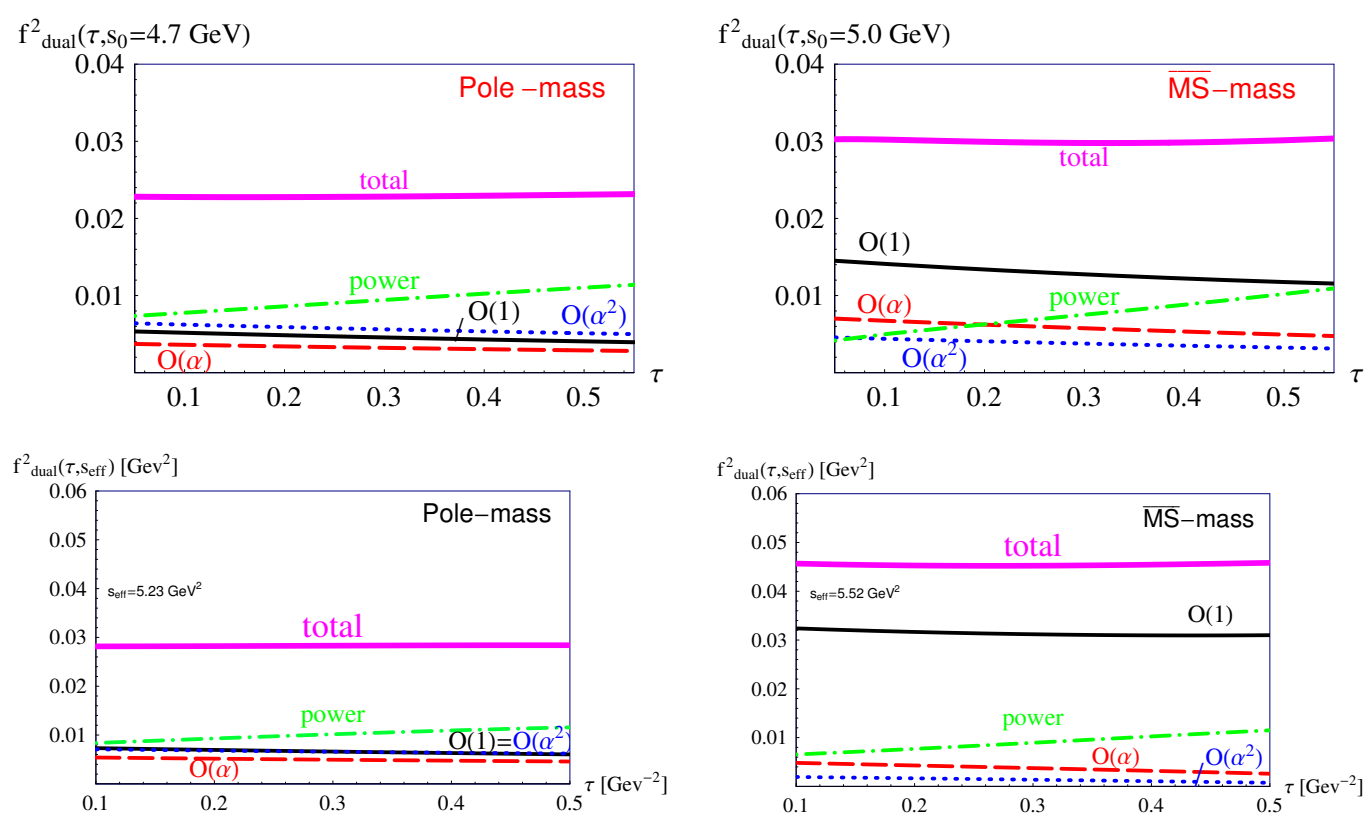

Figure 1. Hierarchy of the OPE contributions to the dual decay constants, $f_{\text {dual }}(\tau)$, for both charmed pseudoscalar meson $D$ (top row) and charmed vector meson $D^{*}$ (bottom row), as obtained in the pole-mass (left column) and the $\overline{M S}$-mass (right column) renormalization scheme, for fixed threshold called $s_{0}$ in the $D$ case and $s_{\text {eff }}$ in the $D^{*}$ case. 


\section{Topical issue: Maximal perturbative convergence [12-14]}

Within perturbation theory, each of the coefficients of the various local operators in the OPE is derived in the shape of a series in powers of the strong coupling, $\alpha_{\mathrm{s}}(\mu)$. In particular, the coefficient multiplying the unit operator ends up in the spectral density, presently determined to three-loop $\left(\alpha_{\mathrm{s}}^{2}\right) \operatorname{order}[15,16]$ :

$$
\rho\left(s, m_{c}, \mu\right)=\rho_{0}\left(s, m_{c}\right)+\frac{\alpha_{\mathrm{s}}(\mu)}{\pi} \rho_{1}\left(s, m_{c}\right)+\frac{\alpha_{\mathrm{s}}^{2}(\mu)}{\pi^{2}} \rho_{2}\left(s, m_{c}, \mu\right)+\cdots .
$$

The rate of convergence of perturbative findings is sensitive to the renormalization scheme defining the $c$-quark's mass. In this respect, using its $\overline{\mathrm{MS}}$ running mass $m_{c}=\bar{m}_{c}\left(\bar{m}_{c}\right)=(1275 \pm 25) \mathrm{MeV}$ is superior to adopting its pole mass $m_{c}=\stackrel{\circ}{m}_{c}=1699 \mathrm{MeV}$, being related by means of given expressions $r_{1,2}$ [17]:

$$
\bar{m}_{c}(\mu)=\stackrel{\circ}{m}_{c}\left(1+\frac{\alpha_{\mathrm{s}}(\mu)}{\pi} r_{1}+\frac{\alpha_{\mathrm{s}}^{2}(\mu)}{\pi^{2}} r_{2}+\cdots\right) .
$$

Inspecting Fig. 1, the gain in perturbative credibility is evident and visibly larger for the vector mesons.

\section{Topical issue: Renormalization-scale dependence [12-14]}

Needless to say, exact correlation functions do not depend on any renormalization scale(s) $\mu$. However, due to practically inevitable truncations to finite-order perturbative expansions or to finite-dimensional vacuum condensates, spectral densities and power corrections and thus predicted hadronic features do. Defining an average $\bar{\mu}$ of the renormalization scale $\mu$ by requiring $f_{\text {dual }}(\bar{\mu})=\left\langle f_{\text {dual }}(\mu)\right\rangle$, such unphysical decay-constant sensitivity to $\mu$ is more pronounced for vector than for pseudoscalar mesons, see Fig. 2:

$$
\begin{aligned}
f_{D}(\mu) & =208.3 \mathrm{MeV}\left[1+0.06 \log (\mu / \bar{\mu})-0.11 \log ^{2}(\mu / \bar{\mu})+0.08 \log ^{3}(\mu / \bar{\mu})\right], \\
f_{D_{s}}(\mu) & =246.0 \mathrm{MeV}\left[1+0.01 \log (\mu / \bar{\mu})-0.03 \log ^{2}(\mu / \bar{\mu})+0.04 \log ^{3}(\mu / \bar{\mu})\right], \\
f_{D^{*}}(\mu) & =252.2 \mathrm{MeV}\left[1+0.233 \log (\mu / \bar{\mu})-0.096 \log ^{2}(\mu / \bar{\mu})+0.17 \log ^{3}(\mu / \bar{\mu})\right], \\
f_{D_{s}^{*}}(\mu) & =305.5 \mathrm{MeV}\left[1+0.124 \log (\mu / \bar{\mu})+0.014 \log ^{2}(\mu / \bar{\mu})-0.034 \log ^{3}(\mu / \bar{\mu})\right] .
\end{aligned}
$$

Table 2 tells us that the averages $\bar{\mu}$ are somewhat larger for the vector than for the pseudoscalar mesons.
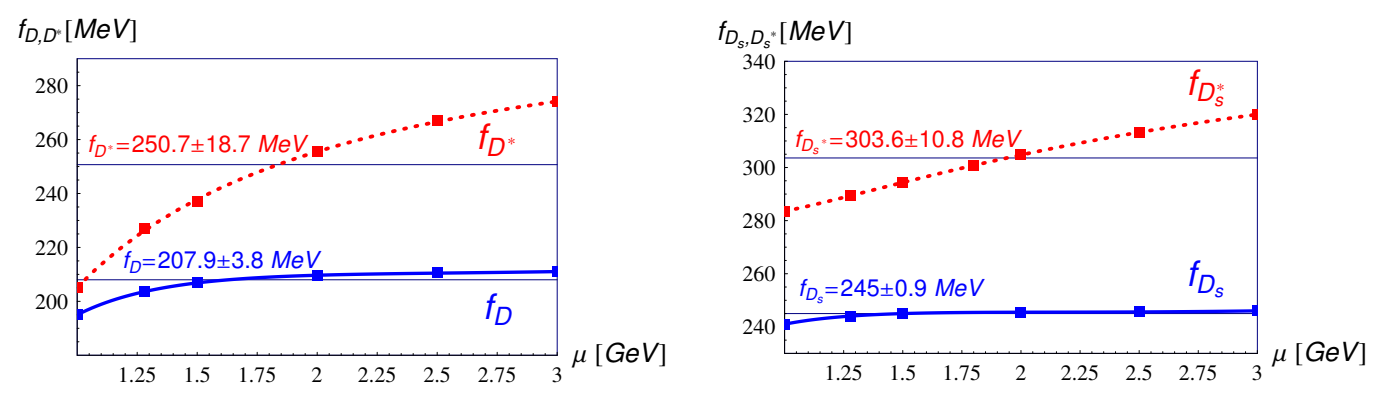

Figure 2. Dependences on the renormalization scale $\mu$ of our QCD sum-rule findings, for the dual decay constants of the charmed non-strange mesons $D$ and $D^{*}\left(f_{D^{(*)}}\right.$, left), and the charmed strange mesons $D_{s}$ and $D_{s}^{*}\left(f_{D_{s}^{(*)}}\right.$, right). 
Table 2. Numerical values of the average renormalization scales $\bar{\mu}$ for the charmed-meson dual decay constants.

\begin{tabular}{lcccc}
\hline \hline Meson & $D$ & $D_{s}$ & $D^{*}$ & $D_{s}^{*}$ \\
\hline $\bar{\mu}(\mathrm{GeV})$ & 1.62 & 1.52 & 1.84 & 1.94 \\
\hline \hline
\end{tabular}

\section{Observations, outcomes, and conclusions}

This simultaneous scrutiny of QCD sum-rule predictions for the decay constants of the charmed vector $[14]$ and pseudoscalar $[12,13]$ mesons discloses, not surprisingly, both similarities and dissimilarities: With respect to the perturbative convergence of the extraction procedures, both types of mesons prefer, beyond doubt, the use of the $\overline{M S}$ definition for the heavy quark's mass. The effects of this are important for vector and pseudoscalar mesons. For both types of mesons, the calculated central values relying on the $\overline{\mathrm{MS}}$ mass are significantly larger than those emerging from the pole mass. Pseudoscalar mesons do not seem to care too much about the precise value of the renormalization scale $\mu$, whereas its impact on the vector mesons is not negligible for their OPE-related errors. Our results for the decay constants are

$$
\begin{aligned}
f_{D} & =\left(206.2 \pm 7.3_{\mathrm{OPE}} \pm 5.1_{\mathrm{syst}}\right) \mathrm{MeV}, & & f_{D_{s}}=\left(245.3 \pm 15.7_{\mathrm{OPE}} \pm 4.5_{\mathrm{syst}}\right) \mathrm{MeV} \\
f_{D^{*}} & =\left(252.2 \pm 22.3_{\mathrm{OPE}} \pm 4_{\mathrm{syst}}\right) \mathrm{MeV}, & & f_{D_{s}^{*}}=\left(305.5 \pm 26.8_{\mathrm{OPE}} \pm 5_{\mathrm{syst}}\right) \mathrm{MeV}
\end{aligned}
$$

\section{References}

[1] M. A. Shifman, A. I. Vainshtein, and V. I. Zakharov, Nucl. Phys. B 147, 385 (1979).

[2] W. Lucha, D. Melikhov, and S. Simula, Phys. Rev. D 76, 036002 (2007), arXiv:0705.0470 [hep$\mathrm{ph}$.

[3] W. Lucha, D. Melikhov, and S. Simula, Phys. Lett. B 657, 148 (2007), arXiv:0709.1584 [hepph].

[4] W. Lucha, D. I. Melikhov, and S. Simula, Phys. Atom. Nucl. 71, 1461 (2008).

[5] W. Lucha, D. Melikhov, and S. Simula, Phys. Lett. B 671, 445 (2009), arXiv:0810.1920 [hep$\mathrm{ph}]$.

[6] D. Melikhov, Phys. Lett. B 671, 450 (2009), arXiv:0810.4497 [hep-ph].

[7] W. Lucha, D. Melikhov, and S. Simula, Phys. Rev. D 79, 096011 (2009), arXiv:0902.4202 [hep$\mathrm{ph}]$.

[8] W. Lucha, D. Melikhov, and S. Simula, J. Phys. G 37, 035003 (2010), arXiv:0905.0963 [hep-ph].

[9] W. Lucha, D. Melikhov, and S. Simula, Phys. Lett. B 687, 48 (2010), arXiv: 0912.5017 [hep-ph].

[10] W. Lucha, D. I. Melikhov, and S. Simula, Phys. Atom. Nucl. 73, 1770 (2010), arXiv:1003.1463 [hep-ph].

[11] W. Lucha, D. Melikhov, H. Sazdjian, and S. Simula, Phys. Rev. D 80, 114028 (2009), arXiv: 0910.3164 [hep-ph].

[12] W. Lucha, D. Melikhov, and S. Simula, J. Phys. G 38, 105002 (2011), arXiv:1008.2698 [hep-ph].

[13] W. Lucha, D. Melikhov, and S. Simula, Phys. Lett. B 701, 82 (2011), arXiv:1101.5986 [hep-ph].

[14] W. Lucha, D. Melikhov, and S. Simula, Phys. Lett. B 735, 12 (2014), arXiv:1404.0293 [hep-ph].

[15] K. G. Chetyrkin and M. Steinhauser, Phys. Lett. B 502, 104 (2001), arXiv:hep-ph/0012002.

[16] K. G. Chetyrkin and M. Steinhauser, Eur. Phys. J. C 21, 319 (2001), arXiv:hep-ph/0108017.

[17] M. Jamin and B. O. Lange, Phys. Rev. D 65, 056005 (2002), arXiv:hep-ph/0108135. 